\title{
GGS Prize 2012
}

The Editors and Advisory Board of Genes \& Genetic Systems are pleased to announce that the GGS Prize 2012 will be awarded to the authors for their paper:

trans-translation-mediated tight regulation of the expression of the alternative ribosome-rescue factor ArfA in Escherichia coli

by Yuhei Chadani, Emi Matsumoto, Hiroaki Aso, Takeo Wada, Kazuhiro Kutsukake, Shizuyo Sutou and Tatsuhiko Abo, published in Genes \& Genetic Systems (2011) 86: 151-163.

This paper was selected by the editors and the members of the advisory board of Genes \& Genetic Systems as one of the best articles published in Genes \& Genetic Systems in 2010 and 2011.

Arguments supporting this decision were:

"This paper provided evidence supporting the existence of a novel system for avoiding accumulation of stalled ribosomes when the mRNA does not possess a stop codon. The novel system proposed here is quite interesting and the logic in the text is easy to follow and to understand for non-specialists in this field."

\section{GGS Prize 2012}

The Editors and Advisory Board of Genes \& Genetic Systems are pleased to announce that the GGS Prize 2012 will be awarded to the authors for their paper:

\section{Identification of an evolutionarily conserved, functional noncoding element regulated by Six1 homeoprotein}

by Yongsu Jeong and Sangtaek Oh, published in Genes \& Genetic Systems (2010) 85: 233-240.

This paper was selected by the editors and the members of the advisory board of Genes \& Genetic Systems as one of the best articles published in Genes \& Genetic Systems in 2010 and 2011.

Arguments supporting this decision were:

"This study found a novel conserved non-coding regulatory sequence, SRE1, to which a homeobox protein Six1 binds. This sequence regulates expression of target genes of Six1 in cranial and spinal ganglia in mouse embryos. The sequence is well conserved from mouse to fish. Experiments and analyses from both molecular and evolutionary viewpoints are integrated to show clearly and comprehensively how this regulatory system works." 